\title{
Hydroxyapatite/NELL-I Nanoparticles Electrospun Fibers for Osteoinduction in Bone Tissue Engineering Application
}

\author{
Hualei Song ${ }^{1} *$ \\ Yuntao Zhang ${ }^{2}$,* \\ Zihan Zhang ${ }^{2, *}$ \\ Shijiang Xiong ${ }^{3}$ \\ Xiangrui $\mathrm{Ma}^{2}$ \\ Yourui $\mathrm{Li}^{2}$ \\ 'Department of Laboratory, Binzhou \\ Medical University Hospital, Binzhou, \\ 256603, People's Republic of China; \\ ${ }^{2}$ Department of Stomatology, Binzhou \\ Medical University Hospital, Binzhou, \\ 256603, People's Republic of China; \\ ${ }^{3}$ Shandong Provincial Key Laboratory of \\ Oral Tissue Regeneration, School of \\ Stomatology, Shandong University, Jinan, \\ 2500 12, People's Republic of China
}

*These authors contributed equally to this work
Correspondence: Yourui Li, Department of Stomatology, Binzhou Medical University Hospital, 66I\#, 2nd Huanghe Road, Binzhou, 256603,

Shangdong Province, People's Republic of China

Tel +865433256715

Email carylee37@qq.com
Background: As commonly bone defect is a disease of jaw that can seriously affect implant restoration, the bioactive scaffold can be used as potential systems to provide effective repair for bone defect.

Purpose: A osteoinductive bone tissue engineering scaffold has been prepared in order to explore the effect of bioactive materials on bone tissue engineering.

Methods: In this study, NELL-1 nanoparticles (Chi/NNP) and nano hydroxyapatite were incorporated in composite scaffolds by electrospinning and characterized using TEM, SEM, contact angle, tensile tests and in vitro drug release. In vitro biological activities such as MC3T3-E1 cell attachment, proliferation and osteogenic activity were studied.

Results: With the addition of nHA and nanoparticles, the fiber diameter of PCL/BNPs group, PCL/NNPs group and PCL/nHA/NNPs group was significantly increased. Moreover, the hydrophilic hydroxyl group and amino group presented in nHA and nanoparticles had improved the hydrophilicity of the composite fibers. The composite electrospun containing Chi/NNPs can form a double protective barrier which can effectively prolong the release time of NELL-1 growth factor. In addition, the hydroxyapatite/NELL-1 nanoparticles electrospun fibers can promote attachment, proliferation, differentiation of MC3T3-E1 cells and good cytocompatibility, indicating better ability of inducing osteogenic differentiation.

Conclusion: A multi-functional PCL/nHA/NNPs composite fiber with long-term bioactivity and osteoinductivity was successfully prepared by electrospinning. This potential composite could be used as scaffolds in bone tissue engineering application after in vivo studies.

Keywords: polycaprolactone, nanoscaffold, MC3T3-E1 cells, osteogenic activity

\section{Introduction}

Bone defect in jaw is a serious medical disease that can cause significant problem in the dental implant restoration. Traditionally bone defect can be treated by different techniques, such as autogenous bone transplantation, allogeneic bone transplantation and distraction osteogenesis, et al. ${ }^{1}$ However, these techniques can be limited due to secondary injury, poor plasticity, immune rejection, high technical requirements, high price and limited osteogenic capacity. In recent years, bone tissue engineering has drawn significant interest in bone defect treatment due to its unique advantages: abundant tissue sources and no secondary infection. ${ }^{2}$ The scaffold materials which can play an important role in bone tissue engineering should be endowed with some characters including it being with three-dimensional porous interconnected structure, good biocompatibility, degradability, being effectively 
loaded and continuously released growth factors. ${ }^{3-6}$ Bone biochemical microenvironment, which mainly consist of ECM molecules, signal molecules and other non-signal soluble factors, can constitute a complex network system for promoting bone reconstruction. As reported, bone biochemical microenvironment is not only the biological basis of bone tissue engineering, but also for the design of scaffold materials and the selection of bioactive factors. Therefore, how to construct an ideal scaffold in accordance with the above principles has become an important area of research, in which the osteogenic activity of scaffold can be considered as a pivotal important factor, and how to confer the scaffold with these properties is being studied. $^{3}$

Polycaprolactone (PCL) is a bioresorbable material with good biocompatibility, and plays an important role in guiding bone regeneration. ${ }^{7}$ At present, polycaprolactone can be fabricated by electrospinning technology into nanofiber scaffold with good biocompatibility, good mechanical strength, porous interconnected structure, high specific surface area, capacity of being loaded with variety of growth factors. What is more important is that these composite scaffolds can simulate the structure of bone natural extracellular matrix. ${ }^{8}$ However, PCL can be restricted its application in bone regeneration due to its weak bioactivity and osteoinductivity. ${ }^{9}$ Nano hydroxyapatite (nHA) as the main inorganic component of bone matrix has a good affinity with adhesion protein and plays an important role in the differentiation and mineralization of osteoblasts, which can provide the composite scaffolds with an effective osteogenictivity. ${ }^{10-13}$ Some research has indicated that the addition of nHA can effectively improve the bioactivity and osteoinductivity of PCL, which can extend its applications in biomedical field. ${ }^{14}$ Ghorbani FM have successfully prepared PCL/nHA composite as scaffolds for bone regeneration, and the results showed that the composite scaffolds could promote osteogenesis and improve bone regenerative ability. ${ }^{15}$ Nevertheless, some growth factors are needed to participate in bone regeneration, and improve the bioactivity and osteogenictivity of PCL. ${ }^{16}$ Therefore, improving the osteogenictivity of PCL/nHA composite fiber has become an urgent need.

There are a variety of signal molecules participating in bone regeneration, among which NELL-1 protein as a new growth factor has attracted more and more attention. NELL-1 located in the downstream of Cbfa1/Runx 2 can participate in the recruitment, growth, proliferation, differentiation, mineralization of osteoblasts and the interaction between cells and intercellular matrix. ${ }^{17}$ What is more important is that NELL-1 protein can induce more dense bone formation but not induce ectopic osteogenesis in muscle due to its specific osteogenictivity. ${ }^{18}$

In our previous study, we have successfully prepared chitosan based-nanoparticles loaded with NELL-1 (Chi/ NNP) by desolvation technique and electrostatic selfassembly. In chitosan based-nanoparticles, chitosan shell was coated on bovine serum albumin (BSA) microsphere loaded with NELL-1 in order to stabilize BSA microsphere; thus, the nanoparticle can slowly release the NELL-1 and maintain the bioactivity of NELL-1. ${ }^{19}$

Based on the above understanding, the purpose of this study was that chitosan based-nanoparticles were incorporated into electrospun scaffolds loaded with nano hydroxyapatite by electrospinning technology, which can construct a double barrier for sustained-release of NELL-1 in order to obtain a better controlled release effect and endow scaffold materials with better osteogenic activity (Figure 1).

\section{Materials and Methods}

\section{Materials and Instruments}

NEL-like molecule 1 (NELL-1), Polycaprolactone (PCL; average Mn 80,000), Bovine Serum Albumin (BSA), Chitosan (medium molecular weight), Fluorescein Isothiocyanate (FITC) and 4',6-diamidino-2-phenylindole (DAPI) were purchased from Sigma (St. Louis, MO, USA). Acetic acid (AA), Absolute alcohol, N. N-Dimethylformamide (DMF), Dichloromethane (DCM) and all chemicals were of analytical reagent grade, without further purification and purchased from Sinopharm chemical reagent Co., Ltd. China. Scanning electron microscope (SEM, Evo LS15,) was from Carl Zeiss, Germany. Transmission electron microscope (TEM, HT7700) was from Hitachi, Japan. Universal mechanical test instrument was from Jinan Runrui Technology Co., Ltd., China. Water contact angle analysis instrument was from TianJin Precision Instrument Co., Ltd., China. Microplate reader was from Thermo Fisher Scientific, US and Confocal laser scanning microscope (CLSM, Leica TCS Sp5 II) was from Leica, Germany.

\section{Preparation of Chitosan Based-Nanoparticle Loaded with NELL-I}

Chitosan-based nanoparticle loaded with NELL-1 (Chi/ NNPs) was produced by desolvation method and electrostatic self-assembly technique according to our previous 


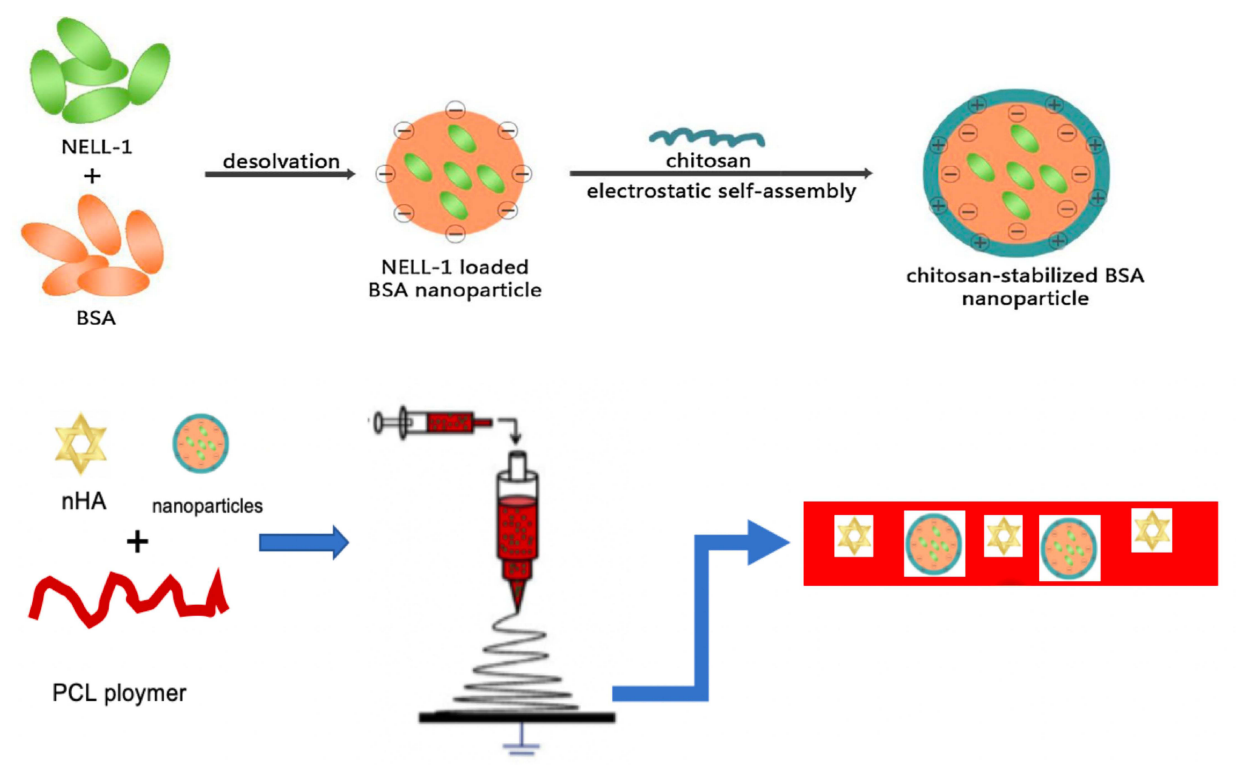

Figure I Schematic illustration of the fabrication of composite electrospun scaffolds loaded with chitosan-based nanoparticles and $\mathrm{nHA}$. Abbreviations: nHA, nano hydroxyapatite; PCL, polycaprolactone; BSA, bovine serum albumin.

reports. ${ }^{19}$ Briefly, BSA (100 $\left.\mathrm{mg}\right)$ and $160 \mu \mathrm{L}$ stock solution $\left(50 \mu \mathrm{g} / 100 \mu \mathrm{L}\right.$ in phosphate buffer at $\left.20^{\circ} \mathrm{C}\right)$ of NELL-1 were dissolved in $10 \mathrm{~mL}$ deionized water, and then $40 \mathrm{~mL}$ of ethanol was then pumped into the $1 \%$ BSA solution using a micro syringe at $0.5 \mathrm{~mL} / \mathrm{min}$ under continual stirring overnight in order to form aggregate. Then, $40 \mathrm{~mL}$ of chitosan solution was added to the mixture at $0.5 \mathrm{~mL} / \mathrm{min}$ speed. The newly formed mixture was stirred for $8 \mathrm{~h}$ to yield the chitosan based-nanoparticle loaded with NELL-1 (Chi/NNPs). The chitosan-based nanoparticle loaded without NELL-1 (Chi/BNPs) were also prepared according to the procedure described above without addition of NELL-1. These chitosan nanoparticles were collected by centrifugation at $12,000 \mathrm{rpm}$ for $20 \mathrm{~min}$. Afterwards, the supernatant was discarded and the precipitated were dispersed in 30/70 distilled water/ethanol (v/ v) solution. These purification steps were repeated 3 times. The white precipitated microspheres were then acquired and stored for future use.

\section{Preparation of Electrospun Scaffolds}

To prepare the electrospinning solution for PCL fiber (PCL), $2.4 \mathrm{~g}$ of PCL was dissolved into $5 \mathrm{~mL}$ of DMF and $7.5 \mathrm{~mL}$ of DCM at room temperature. To prepare the electrospinning solution loaded with chitosan-based nanoparticle (PCL/ BNPs, PCL/NNPs), the nanospheres (BNPs or NNPs) were dissolved in $2.5 \mathrm{~mL}$ of DMF solution, and then $1.5 \mathrm{~g}$ of PCL was dissolved into $7.5 \mathrm{~mL}$ of DCM. Then, the DMF solution with BNPs or NNPs was added drop by drop into DCM solution under magnetic stirring for at least 8 hours. To prepare the electrospinning solution loaded with nanohydroxyapatite (PCL/nHA), nano-hydroxyapatite (30\%, wt/ wt), was added into PCL solution under magnetic stirring for at least 8 hours until it was completely mixed. To prepare the electrospinning solution loaded with nHA and chitosanbased nanoparticles (PCL/nHA/NNPs), according to the procedure descripted above, DMF solution dispersed with nanospheres was added drop by drop into DCM solution containing nHA under magnetic stirring for at least $8 \mathrm{~h}$ until it was completely mixed. Details of solution preparation for electrospinning can be shown in Table 1.

The electrospinning solution was placed into a $30 \mathrm{~mL}$ plastic syringe equipped with a medical-grade $6^{\#}$ stainless steel needle. The specific electrospun conditions were as follows: the high voltage was $25 \pm 0.5 \mathrm{kV}$, the flow rate of solution controlled by the micro injection pump was $3 \mathrm{~mL} / \mathrm{h}$, and the receiving distance was $20 \mathrm{~cm}$. All the electrospun films were collected at room temperature and dried in a vacuum drying oven.

\section{Characterization}

Transmission Electron Microscope (TEM) characterization

All samples were deposited on the carbon film copper network within 5 seconds during electrospinning. After 
Table I Details of Solution Preparation for Electrospinning and Nanoparticles for All Polymers Including Molecular Weight (Mw), Concentration w/w (C), Solvents, Stirring Time $(t)$, The Rotation Speed of Stirrer $\left(V_{r}\right)$, and Temperature of the Hot Plate During Mixing

\begin{tabular}{|l|l|l|l|l|l|l|l|}
\hline Polymer & Supplier & $\mathbf{M w}\left(\mathbf{g} \cdot \mathbf{m o l}^{-\mathbf{1}}\right)$ & $\mathbf{C ~ ( \% )}$ & Solvents & $\boldsymbol{t}(\mathbf{h})$ & $\mathbf{V r}[\mathbf{r p m}]$ & $\mathbf{T}\left({ }^{\circ} \mathbf{C}\right)$ \\
\hline PCL & Sigma-Aldrich & 80,000 & 75.47 & DCM & 4 & 800 & 25 \\
\hline Chi/NNPs & - & - & 4.23 & DMF & 8 & 800 & 25 \\
\hline Chi/BNPs & - & - & 4.23 & DMF & 8 & 800 & 25 \\
\hline Hydroxyapatite & Macklin, China & 1004 & 30 & DCM & $\geq 8$ & 1200 & 25 \\
\hline Chitosan & Sigma-Aldrich & $50,000-190,000$ & 0.15 & AA & 1 & 400 & 25 \\
\hline
\end{tabular}

Abbreviations: PCL, polycaprolactone; Chi/NNPs, chitosan based-nanoparticles loaded with NELL-I; Chi/BNPs, chitosan based-nanoparticle loaded without NELL-I; AA, acetic acid; DMF, N.N-Dimethylformamide; DCM, dichloromethane.

being dried, all samples were observed by field emission transmission electron microscopy (HT7700, Hitachi, Japan) at an acceleration voltage of $80 \mathrm{kV}$.

\section{Scanning Electron Microscope (SEM) Characterization}

All groups of electrospun fibers were cut into $4.5 \mathrm{~mm} * 4.5 \mathrm{~mm}$ in size and fixed on the metal stage with conductive adhesive. After being sprayed with gold, all samples were observed by using SEM (Evo LS15, Carl Zeiss, Germany) with electron acceleration voltage of 20 $\mathrm{kV}$. All photos captured by SEM were analyzed by using Image Pro Plus 6.0 software.

\section{Tensile Strength}

All groups of electrospun materials were cut into rectangle strips $(5 \mathrm{~cm} * 2 \mathrm{~cm})$ with thickness of $0.5 \mathrm{~mm}$. The universal testing machine was used to test the tensile strength at the same parameters as follows: the tensile rate was $10 \mathrm{~mm} /$ min, the temperature was $20^{\circ} \mathrm{C}$, and the relative humidity was $50 \%$.

\section{Contact Angle Characterization}

A drop of $\mathrm{ddH}_{2} \mathrm{O}(0.5 \mathrm{~mL})$ was dropped on the surface of the electrospun material fixed on the glass slide. And then the digital camera was used to capture the image of the droplet on the surface of the material and then the contact angle was calculated by image processing software. The reported contact angle was the average of 6 measurements.

\section{In vitro Drug Release}

The release kinetic of NELL-1 in electrospun materials was measured in this study. The Human NELL-1 Elisa Kit was used to determine the protein concentration. Briefly, a certain amount of electrospun materials loaded with NELL-1 was placed in glass vials filled with $5 \mathrm{~mL}$ PBS, and incubated at $37^{\circ} \mathrm{C}$. At the scheduled time point (12h,1d,2d,3d,4d,5d,6d,7d,8d,10d,14d, 18d,22d,26d,30d), $1 \mathrm{~mL}$ PBS in glass vials was absorbed and put into another new glass vial, and then $1 \mathrm{~mL}$ new PBS was added to the original glass vial. Nell-1 ELISA kit was used to detect the release of NELL-1 based on the standard curve established in advance and the cumulative release was calculated at preset time point. The release curve of Chi/NNPs microspheres was also determined according to our previous study. ${ }^{19}$

\section{In vitro Cell Tests}

Cell attachment, proliferation, and migration tests were performed using MC3T3-E1 cells (Subclonal line 14, derived from C57BL/6 mice). All groups of electrospun materials were cut into 6-well plate size, disinfected by ultraviolet light in super-clean worktable and fixed with a sterile steel ring in 6-well plate. And then MC3T3-E1 cells with density of $2 \times 10^{4} / \mathrm{cm}^{2}$ were seeded on electrospun materials and cultured in standard cell culture conditions.

\section{Cell Attachment}

After MC3T3-E1 cells were cultured on the scaffold materials for $12 \mathrm{~h}$, the culture medium was removed and the cells were fixed by $2.5 \%$ glutaraldehyde at $4{ }^{\circ} \mathrm{C}$ for $4 \mathrm{~h}$. And then the cells were dehydrated with gradient alcohol $(30 \%, 50 \%, 70 \%, 95 \%, 100 \%, \mathrm{~V} / \mathrm{V})$ for $10 \mathrm{~min}$, respectively. The SEM (Evo LS15, Carl Zeiss, Germany) was used to observe cell attachment on materials after being vacuumdried and gold-sprayed. In addition, the cytoskeleton was labeled by phalloidin-labeled-rhodamine and the cell nucleus was labeled by 4',6-diamidino-2-phenylindole (DAPI). And then confocal laser scanning microscopy (CLSM, Leica TCS Sp5 II, Germany) was used to observe the extension and adhesion of MC3T3-E1 cells on materials. 


\section{CCK-8 Assay}

MC3T3-E1 cells with density of $2 \times 10^{3} /$ well were seeded in 96 well plate where the electrospun material was fixed with sterile steel ring. At the predetermined time points ( 1 day, 4 days, 7 days) the original culture medium was discarded and CCK-8 solution $(10 \mu \mathrm{L})$ was added into 96 well plate, the cells were incubated for $4 \mathrm{~h}$ at $37^{\circ} \mathrm{C}$. The supernatant was carefully absorbed into a new 96 well plate and then detected by Thermo scientific microplate reader at $450 \mathrm{~nm}$ to get absorbance value (OD). A blank group (without cells and materials) and a control group (containing cells without materials) were set up.

\section{Alkaline Phosphatase Assay(ALP)}

MC3T3-E1 cells in a density of $1 \times 10^{5} /$ well were seeded on 24 well plate in which the electrospun material was fixed with sterile steel ring. The medium was changed every 2 days. After the induction solution ( $\alpha$-MEM medium with $10 \%$ FBS, $10-8 \mathrm{~m}$ dexamethasone and $10 \mathrm{mM} \beta$ glycerophosphate $(50 \mathrm{mg} / \mathrm{mL}$ ascorbic acid)) was added, the samples absorbance value (OD) at $405 \mathrm{~nm}$ were tested according to ALP kit instructions at preset time (7 days, 14 days and 21 days) with three multiple holes in each group.

\section{Reverse Transcription-Polymerase Chain Reaction (RT-PCR)}

MC3T3-E1 cells in a density of $1.5 \times 10^{5} /$ well were seeded in 6-well plates in which the electrospun material was fixed with sterile steel ring. The medium was changed every 2 days. After the induction solution was added, the scaffolds with cells were cut and the total RNA was extracted by Trizol at preset time ( 7 days, 14 days and 21 days). RT-PCR was used to detect the expression of osteogenic genes such as OPN when GAPDH was used as a control gene.

\section{Statistical Analysis}

IBM SPSS Statistics 26.0 software was employed for data analysis. All data were presented as mean \pm standard deviation (S.D.). One-way ANOVA analysis was used to compare the mean in different groups. $P<0.05$ was considered statistically significant.

\section{Results and Discussion Morphologies and Properties of Electrospun Scaffolds}

The morphologies of electrospun nanofiber are shown in Figure 2. In all groups, nanofibrous were prepared
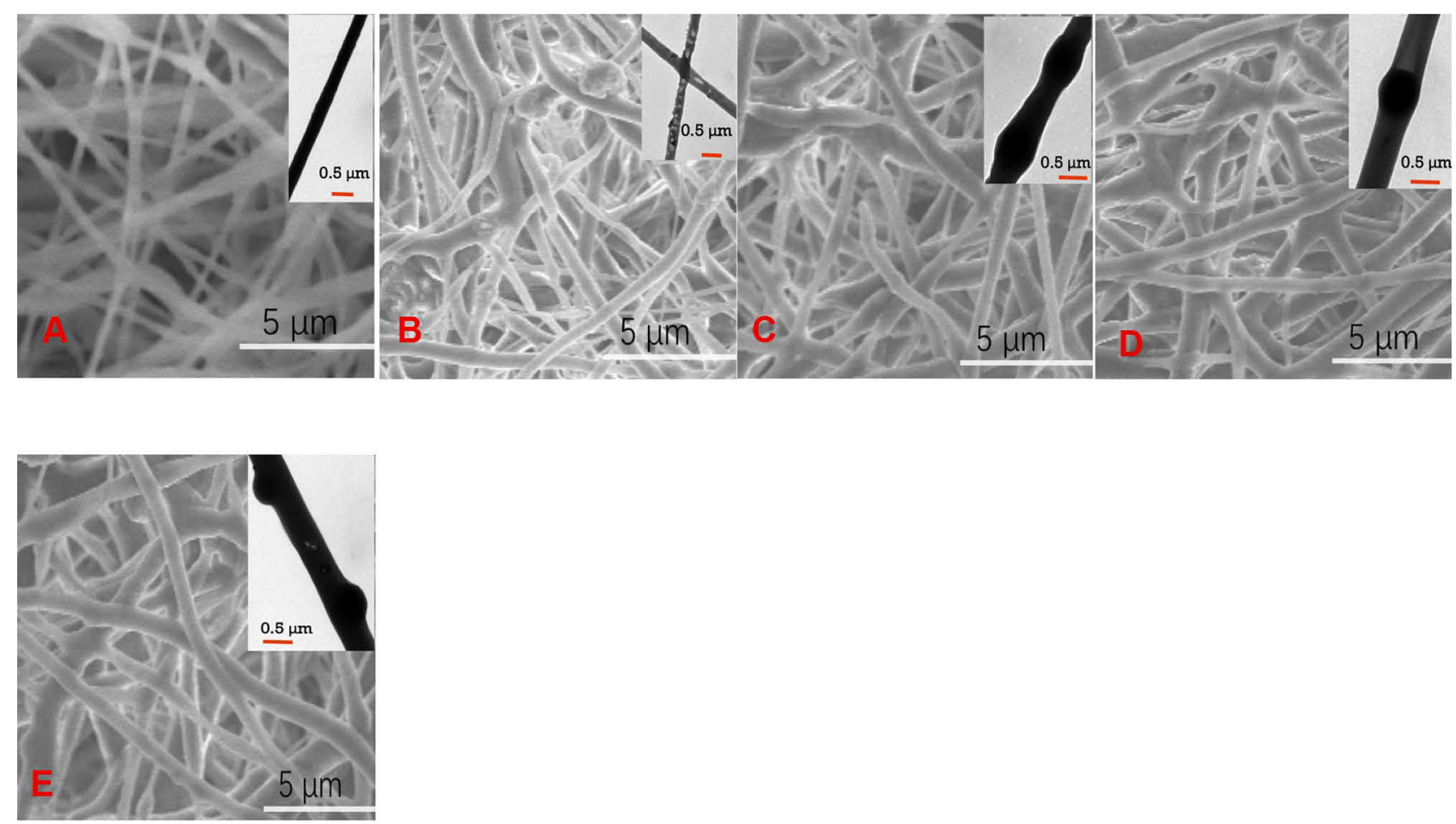

Figure 2 SEM and TEM images of electrospun fibers with TEM images inserted on the top right in each group.

Notes: (A) PCL group; (B) PCL/nHA group; (C) PCL/BNPs group; (D) PCL/NNPs group; (E) PCL/nHA/NNPs group.

Abbreviations: SEM, scanning electron microscope; TEM, transmission electron microscope; PCL, polycaprolactone; nHA, nano hydroxyapatite; NNPs, chitosan basednanoparticles loaded with NELL-I; BNPs, chitosan based-nanoparticle loaded without NELL-I. 


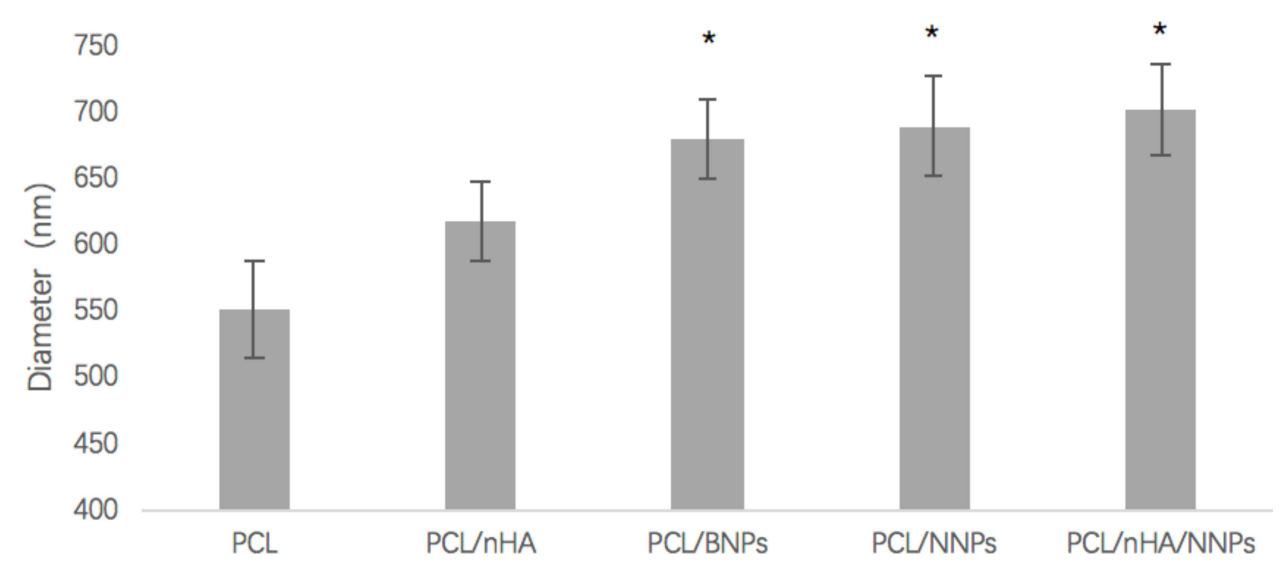

Figure 3 Diameters of electrospinning fiber in different group. Note: $* P<0.05$ compared to the $P C L$ group.

successfully, which presented disordered structure interlaced into a network. The pure PCL nanofiber was uniform with smooth surface, while the nanofibers embedded with nHA had certain agglomeration. The electrospun fiber embedded with nanospheres (BNPs or NNPs) has a slight protrusion which could lead to larger diameter. As shown in Figure 3, the diameters of electrospun nanofibers in PCL group, PCL/nHA group, PCL/BNPs group, $\mathrm{PCL} / \mathrm{NNPs}$ group and PCL/nHA/NNPs group were 550.90 $\pm 37.43 \mathrm{~nm}, \quad 617.18 \pm 30.17 \mathrm{~nm}, 680.23 \pm 29.36 \mathrm{~nm}, 689.36$ $\pm 37.04 \mathrm{~nm}$ and $702.72 \pm 34.24 \mathrm{~nm}$, respectively. Compared with pure PCL group, the fiber diameter of PCL/BNPs group, PCL/NNPs group and PCL/nHA/NNPs group was significantly increased $(P<0.05)$ (Figure 3$)$. The results could be ascribed to the change of solution viscosity and the introduction of nanoparticles and nHA..$^{20,21}$

\section{Tensile Strength and Hydrophilicity}

As shown in Figure 4, the tensile strength in pure PCL group is the highest, reaching $14.98 \pm 1.13 \mathrm{MPa}$. Compared with PCL group, the tensile strength in other four groups was significantly lower $(P<0.05)$, but there was no significant difference between these four groups $(P>0.05)$. With the addition of nHA or nanospheres, the tensile strength of electrospun fibers decreased, which can be ascribed to the addition of nHA or microspheres leading to the decrease of fiber continuity. The results of tensile strength of composite fibers showed sufficient mechanical properties to support proliferation of MC3T3-E1 cells. ${ }^{22,23,24}$

Surface hydrophilicity is a major factor affecting cellular activities of the composite fibers such as adhesion and migration. ${ }^{25}$ Figure 5 shows the hydrophilicity test result of the composite fiber and the contact angles of all groups except

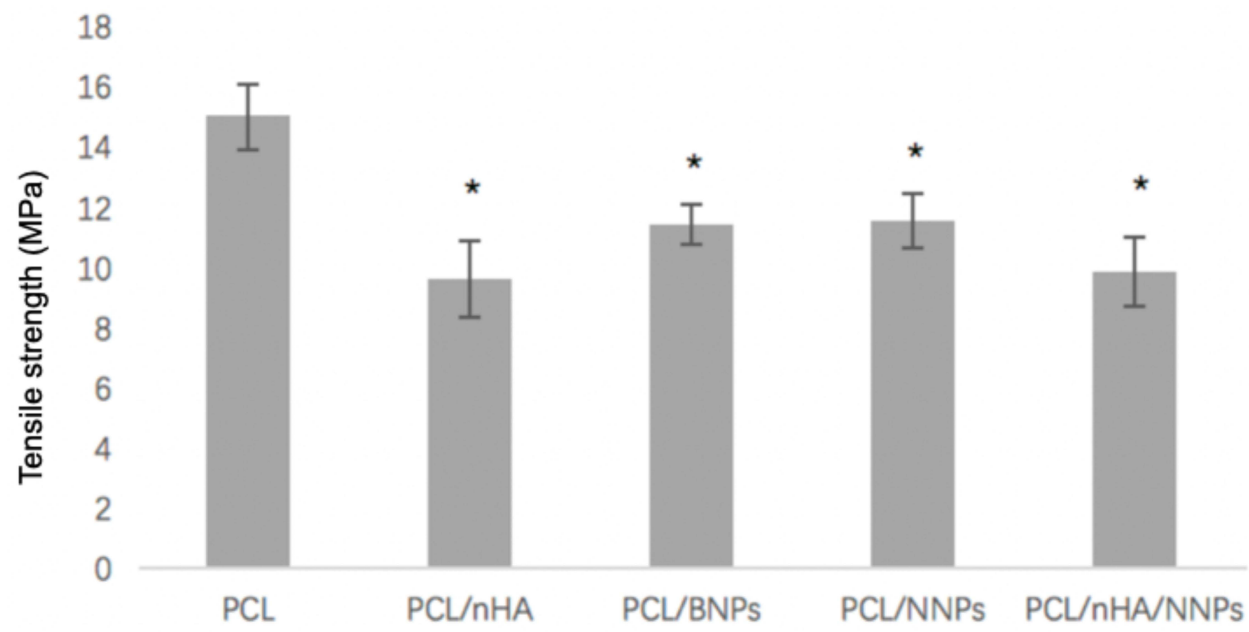

Figure 4 The tensile strength of electrospun fibers in different group. Note: $* P<0.05$ compared to the $P C L$ group. 


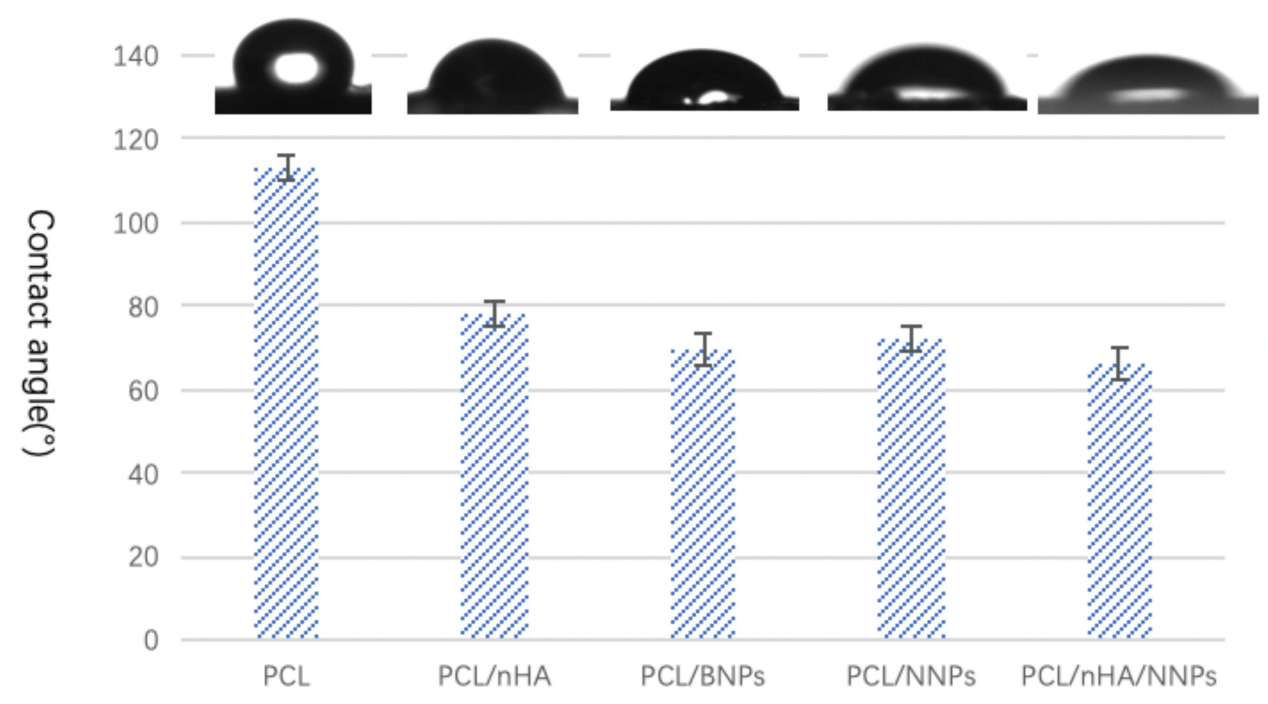

Figure 5 Contact angle of electrospun fibers in different group with images inserted on the top.

PCL group decreased significantly and were less than $90^{\circ}$, indicating that these group showed best hydrophilicity. The improved surface hydrophilicity can be resulted from the hydrophilic hydroxyl group and amino group presented in nHA and nanoparticles in composite fibers. ${ }^{26}$ The improved surface hydrophilicity also can be ascribed to the change of surface roughness due to protrusions of the nanoparticles and nHA, which were confirmed morphologically using SEM and TEM in according with other scholars' results. ${ }^{21,27}$ In addition, hydrophilicity can play a vital role in promoting cell adhesion at an early stage. ${ }^{26}$

\section{Drug Release in vitro}

As shown in Figure 6, the curve of NELL-1 release in pure Chi/NNPs nanoparticles was relatively gentle, and the release rate was $25.02 \pm 4.15 \%$ on the first day, while that of electrospun fibers was more moderate without obvious burst release. There was no statistical difference between PCL/NNPs group $(12.22 \pm 1.68 \%)$ and PCL/nHA/NNPs group (15.49 $\pm 3.11 \%)$ in release rate $(P>0.05)$. More than $95 \%$ of the drug could be significantly sustain-released for about 8 days in Chi/NNPs microsphere group. However, the drug in PCL/NNPs group and $\mathrm{PCL} / \mathrm{nHA} / \mathrm{NNPs}$ group could be released at low concen-

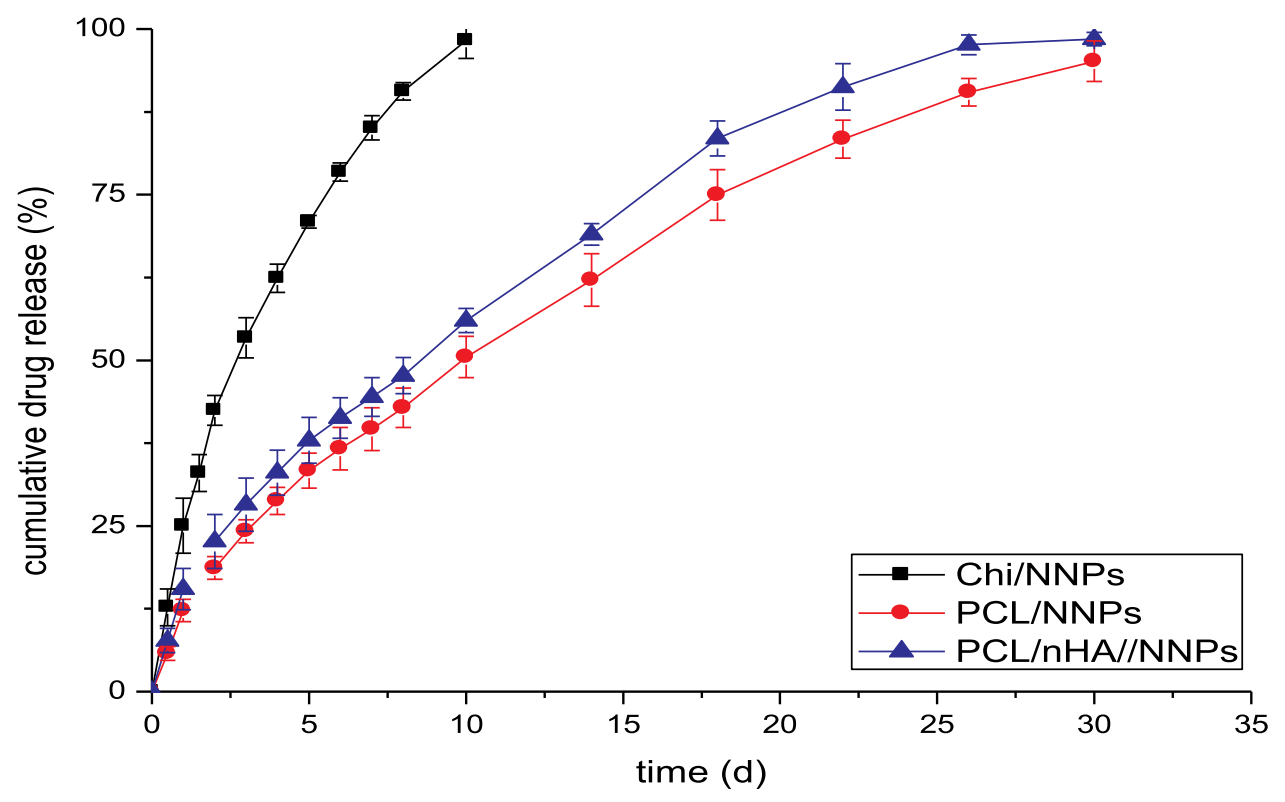

Figure 6 Cumulative release curve of NELL-I growth factor in different samples.

Abbreviations: Chi/NNPs or NNPs, chitosan based-nanoparticles loaded with NELL-I; PCL, polycaprolactone; nHA, nano hydroxyapatite. 
tration for more than 30 days, and the release amount was more than $92 \%$. As shown in Figure 6, the release curve of PCL/NNPs group was similar to that of PCL/nHA/NNPs group. These results indicate that the burst release of drugs can be significantly reduced in electrospun group, which can avoid the side effects of excessive drug release at the initial stage, and prolong the release time of NELL-1 protein.

The structure of chitosan based-nanoparticles embedded in fibers can present the following characteristics: ${ }^{28,30}$ on the one hand, nanofibers can provide space for the nanoparticles embedded in them; on the other hand, nanoparticles and nanofibers provide dual protection for the growth factors/ drugs loaded on the microspheres, which can effectively avoid bad influence on growth factor and maintain good biological activity of growth factors. ${ }^{31-33}$, In other research, the nanoparticles loaded with VEGF and BMP-2 were embedded into the electrospun fiber, the result showed that the release time of growth factor was prolonged, the obvious sudden release was avoided at the initial stage, and at the same time the original biological activity of growth factor could be maintained. $^{34-36}$ In this study, chitosan nanoparticles loaded with NELL-1 were incorporated into electrospinning fibers, the results showed that obvious spherical protrusions were observed in the fiber in TEM images. In other words, double barrier protection for growth factor can significantly delay the release of growth factor Nell-1, ${ }^{37,38}$ which can meet the continuous demand of growth factor in bone tissue regeneration, which is similar to the results of other scholars. ${ }^{20}$
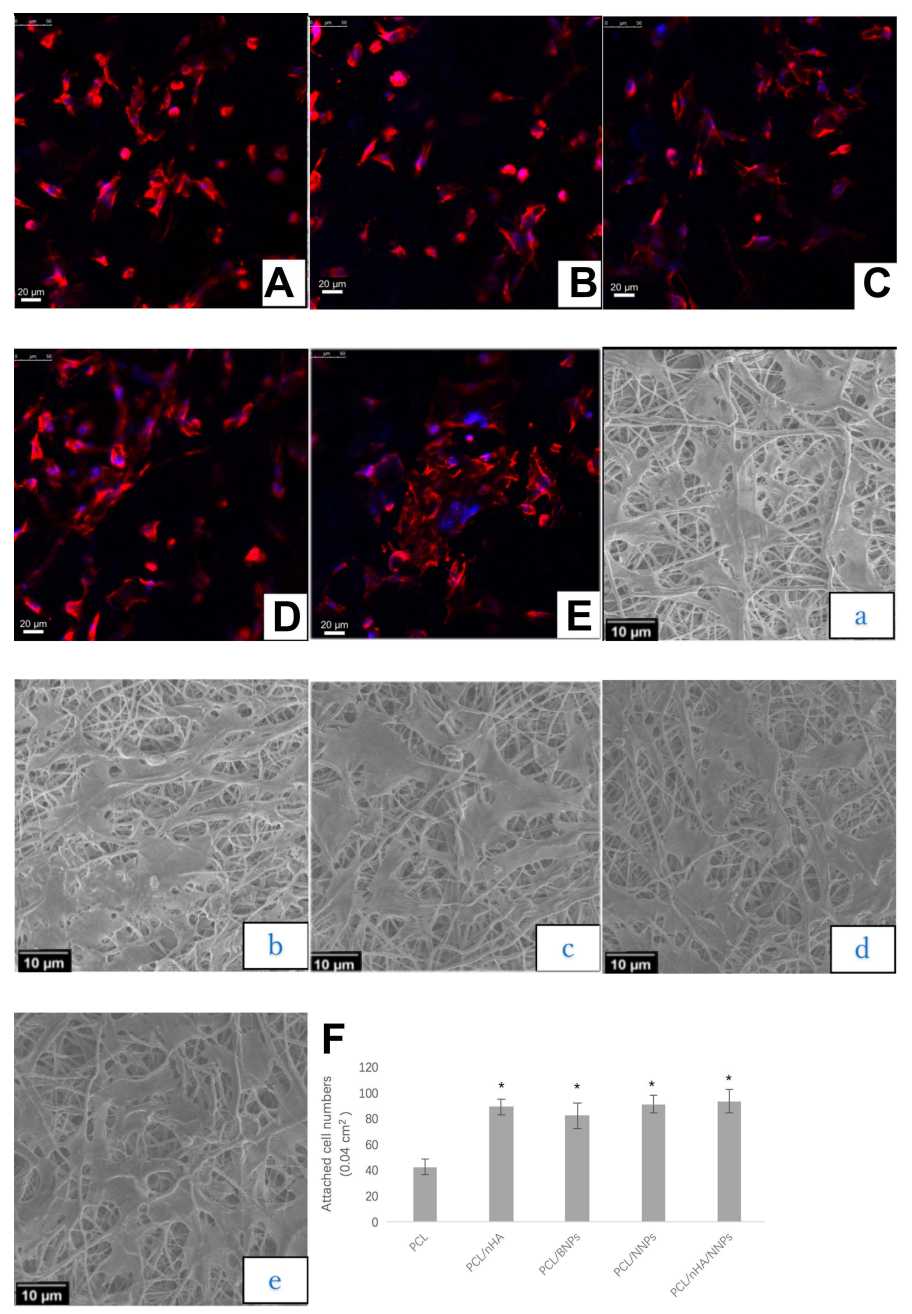

Figure 7 MC3T3-EI cell adhesion number, MC3T3-EI cell adhesion morphology (SEM images), actin cytoskeletal organization and nucleus of cells (CLSM images) after incubation with different samples.

Notes: (A-E), CLSM images; (a-e), SEM images; (A, a) PCL group, (B, b) PCL/nHA group, (C, c) PCL/BNPs group, (D, d) PCL/NNPs group, (E, e) PCL/nHA/NNPs group. (F), MC3T3-EI cell adhesion number after incubation with different samples; $* P<0.05$ compared to the $P C L$ group.

Abbreviations: SEM, scanning electron microscope; CLSM, confocal laser scanning microscope; PCL, polycaprolactone; nHA, nano hydroxyapatite; NNPs, chitosan basednanoparticles loaded with NELL-I; BNPs, chitosan based-nanoparticle loaded without NELL-I. 


\section{In vitro Osteogenic Evaluation of Composite Fiber}

Microscopy investigation allowed us a direct observation of the cells' morphology. MC3T3-E1 cell morphology after 12 hours seeding on the composite fiber scaffolds is shown in Figure 7A-E 7a-e. The results were consistent with CLSM images (Figure 7A-E), in which actin and nucleus of the cytoskeleton were labeled with rhodamine phalloidin and DAPI, respectively, presenting as well spread cells in all groups. As it can be seen from Figure 7, MC3T3-E1 cells were anchored to the surface via filopodia along the electrospun fibers with good adhesion and growth. However, the cells on the composite scaffolds (PCL/nHA group, PCL/ BNPs group, PCL/NNPs group and PCL/nHA/NNPs group) seemed to show better adherence with increased cell numbers (Figure 7F) and better cell-fiber entanglement, perhaps due to the better surface properties with increased hydrophilicity and the presence of the nanoparticles and nHA providing more cell adhesion sites. ${ }^{39}$ Nano hydroxyapatite can induce adhesion, proliferation and differentiation of osteoblasts. Zhang et al prepared composite material loaded with nHA as scaffolds for bone regeneration, and found that the composite scaffolds could promote osteogenesis. ${ }^{40}$ Nastaran et al prepared scaffolds with chitosan and found that chitosan could enhance osteoblast proliferation activity, indicating its better cytocompatibility. ${ }^{28,41}$ NELL-1 can induce MC3T3-E1 cells and promote cell adhesion. ${ }^{29,42}$ As our results showed that the composite scaffolds containing hydroxyapatite and microspheres can show good biocompatibility and significantly promote cell adhesion and growth. Proliferation of MC3T3-E1 cells cultured on the composite scaffolds was evaluated via changes in OD using the CCK-8 kit (Figure 8). On the 1st day of cell culture, the metabolic activity of MC3T3-E1 cells on all groups was similar, and there was no significant difference. On the 4th day of cell culture, the OD value (metabolic activity) of MC3T3-E1 cells on the surface of PCL/NNPs group and PCL/nHA/NNPs group were higher than other groups. The results can be ascribed to the improvement in hydrophilicity of the fiber surface and bioactive functional groups such as -OH and $-\mathrm{NH}_{2}$ in nanoparticles. ${ }^{43}$ The osteogenic activity of MC3T3-E1 cells cultured on the composite scaffolds was assessed via ALP activity (Figure 9). On the 14th days and 21th day, the metabolic activity (OD value) of MC3T3-E1 cells on the surface of PCL/nHA group, PCL/ NNPs group and PCL/nHA/NNPs were higher than other groups. This result might be due to the improvement in hydrophilicity of the fiber surface and the bioactive functional groups in nanoparticles. ${ }^{19,42}$ RT-PCR was used to measure osteogenic activity of MC3T3-E1 cells in different scaffolds (Figure 10). On the 7th day of cell culture, the osteogenic activity (OPN mRNA) of MC3T3-E1 cells on all groups was similar, and there was no significant difference. On the 14th day of cell culture, the expression of OPN mRNA in PCL/nHA group, PCL/NNPs group and PCL/ nHA/NNPs group was significantly higher than that of pure

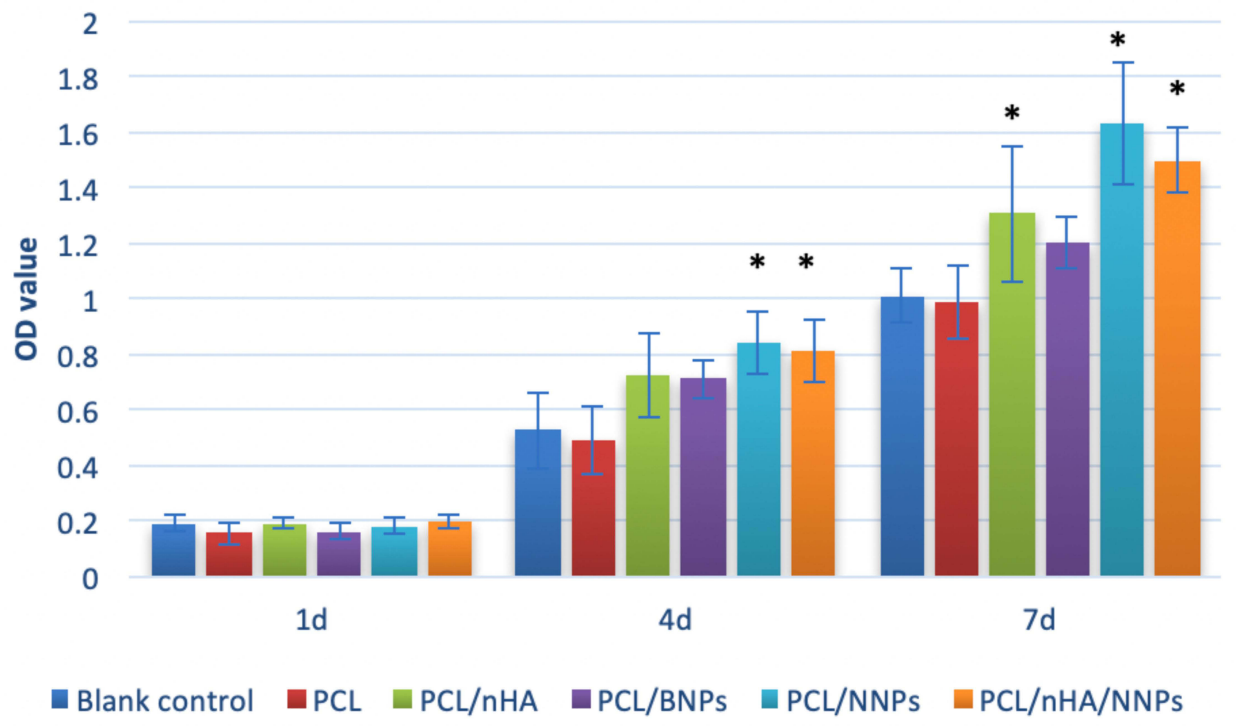

Figure 8 CCK-8 results of MC3T3-EI cells after incubation with different samples for I days, 4 days and 7 days.

Note: $* P<0.05$ compared to the $P C L$ group and blank control.

Abbreviations: d, day; OD, optical density. 


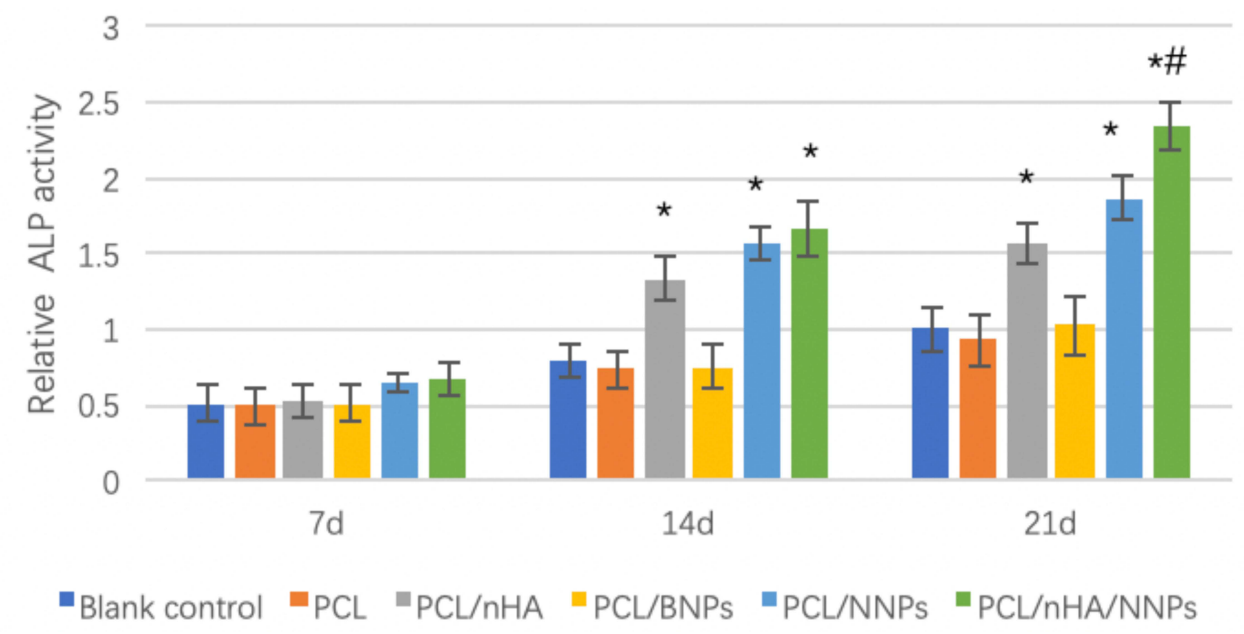

Figure 9 MC3T3-EI cells ALP activity after cultivating for 7 days, 14 days and 21 days with different samples.

Notes: ${ }^{*} P<0.05$ compared to the blank control group, $\mathrm{PCL}$ group and $\mathrm{PCL} / \mathrm{BNPs}$ group. ${ }^{\#} \mathrm{P}<0.05$ compared to $\mathrm{PCL} / \mathrm{nHA}$ group and $\mathrm{PCL} / \mathrm{NNPs}$ group. Abbreviations: d, day; ALP, alkaline phosphatase.

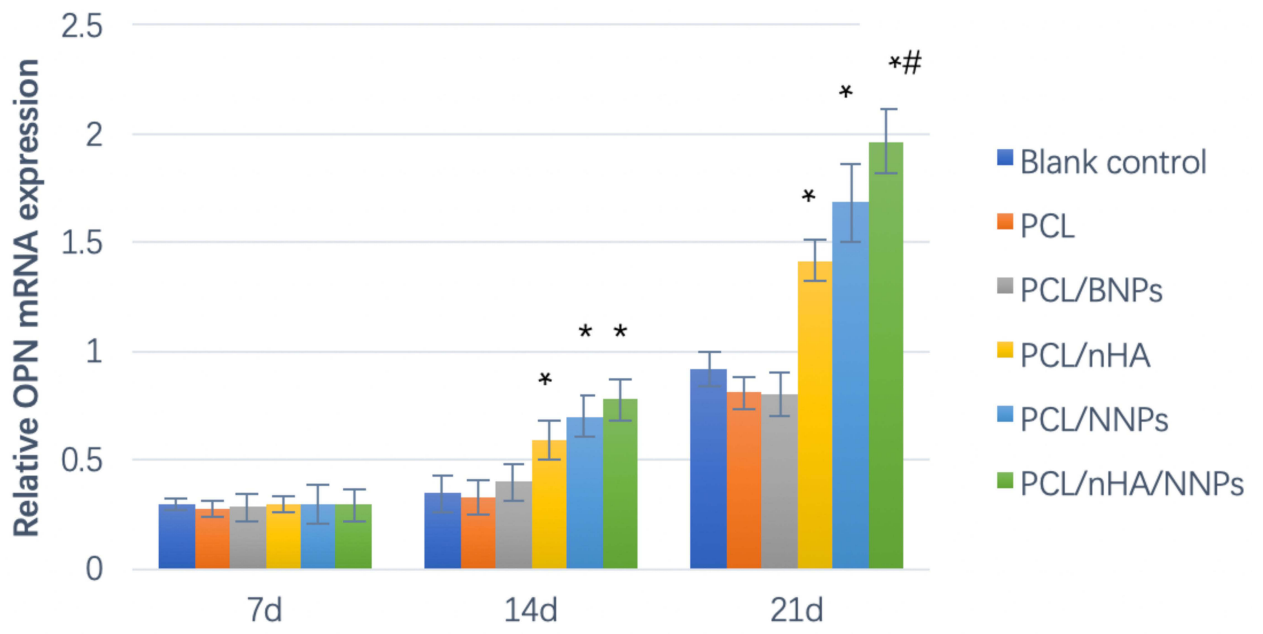

Figure 10 The OPN mRNA expression of MC3T3-EI cells after incubation with different scaffolds for 7 days, 14 days and 21 days. Notes: ${ }^{*} P<0.05$ compared to the Blank control group, $\mathrm{PCL}$ group and $\mathrm{PCL} / \mathrm{BNPs}$ group. ${ }^{\#} \mathrm{P}<0.05$ compared to $\mathrm{PCL} / \mathrm{nHA}$ group. Abbreviations: d, day; OPN, osteopontin.

PCL group and PCL/BNPs group $(P<0.05)$. On the 21st day of cell culture, the osteogenic activity of MC3T3-E1 cells on the surface of PCL/nHA/NNPs fibers was the highest. This might be related to NELL-1 promoting on OPN expression, this result was in accordance with Shen et al results that Nell1 can activate the ERK pathway in pre-osteoblasts. ${ }^{17,44}$

\section{Conclusion}

This study has successfully synthesized chitosan-based nanoparticle loaded with NELL-1 protein (Chi/NNPs) by desolvation method and electrostatic self-assembly technique, and prepared bioactive and osteoinductive PCL/nHA/NNPs composite fiber by electrospinning. The results showed that with the addition of nHA and nanoparticles, the fiber diameter of PCL/BNPs group, PCL/ NNPs group and PCL/nHA/NNPs group was significantly increased. Moreover, the hydrophilic hydroxyl group and amino group presented in nHA and nanoparticles had improved the hydrophilicity of the composite fibers. The composite electrospun containing Chi/NNPs can form double protective barriers which can effectively prolong the release time of NELL-1 growth factor. The PCL/nHA/ 
NNPs composite fiber can promote attachment, proliferation, osteogenic differentiation and maturation of MC3T3E1 cells. The prepared composite electrospun material (PCL/nHA/NNPs) and the protocol proposed for the preparation of composite resulted in potential scaffold with proper mechanical properties, biological properties and osteogenic ability. This potential scaffold could be used for bone tissue engineering application after in vivo studies.

\section{Abbreviations}

PCL, Polycaprolactone; nHA, Nano-Hydroxyapatite; Chi/ NNP, Chitosan based-nanoparticles loaded with NELL-1; Chi/BNPs, Chitosan based-nanoparticle loaded without NELL-1; PCL/BNPs, The electrospun fiber embedded with BNPs; PCL/NNPs, The electrospun fiber embedded with NNPs; PCL/nHA, The electrospun fiber loaded with nHA; PCL/nHA/NNPs, The electrospun fiber embedded with NNPs and nHA; BSA, Bovine Serum Albumin; FITC, Fluorescein Isothiocyanate; DAPI, 4',6-diamidino2-phenylindole; AA, Acetic Acid; DMF, N. N-Dimethylformamide; DCM, Dichloromethane; SEM, Scanning electron microscope; TEM, Transmission electron microscope; CLSM, Confocal laser scanning microscope; ALP, Alkaline phosphatase assay; RT-PCR, Reverse Transcription-Polymerase Chain Reaction.

\section{Ethical Statement}

All MC3T3-E1 cell line procedures were performed in accordance with the Guidelines for Care and Use of Laboratory Cell Line of Binzhou Medical University Hospital and Experiments were approved by the Ethics Committees for Cell Line Experiments of Binzhou Medical University Hospital.

\section{Acknowledgments}

This work was supported by Shandong Medical\&Health Science and Technology Development Project of China (Grant number: 2017WS753, 2017WS231), Natural Science Foundation of Shandong Province of China (Grant number: ZR2018PH023) and Scientific Research Project of Binzhou Medical University of China (Grant number: BY2016KJ06, BY2019KJ35).

\section{Disclosure}

The authors report no conflicts of interest in this work.

\section{References}

1. Amini AR, Laurencin CT, Nukavarapu SP. Bone tissue engineering: recent advances and challenges. Crit Rev Biomed Eng. 2012;40:363-408. doi:10.1615/CritRevBiomedEng.v40.i5.10

2. Agarwal R, García AJ. Biomaterial strategies for engineering implants for enhanced osseointegration and bone repair. Adv Drug Deliv Rev. 2015;94:53-62. doi:10.1016/j.addr.2015.03.013

3. Place ES, Evans ND, Stevens MM. Complexity in biomaterials for tissue engineering. Nat Mater. 2009;8:457-470. doi:10.1038/ nmat2441

4. Stevens MM. Biomaterials for bone tissue engineering. Mater Today. 2008;11:18-25. doi:10.1016/S1369-7021(08)70086-5

5. Singh AV, Gemmati D, Kanase A, et al. Nanobiomaterials for vascular biology and wound management: a review. Veins Lymphatics. 2018;7(2):7196-7209. doi:10.4081/vl.2018.7196

6. Singh AV, Aditi AS, Gade WN, et al. Nanomaterials: new generation therapeutics in wound healing and tissue repair. Curr Nanosci. 2010;6(6):577-586. doi:10.2174/157341310793348632

7. Basar AO, Sadhu V, Turkoglu Sasmazel H. Preparation of electrospun PCL-based scaffolds by mono/multi-functionalized GO. Biomed Mater. 2019;14(4):045012-045027. doi:10.1088/1748-605X/ab2035

8. Li L, Zhou G, Wang Y, et al. Controlled dual delivery of BMP-2 and dexamethasone by nanoparticle-embedded electrospun nanofibers for the efficient repair of critical-sized rat calvarial defect. Biomaterials. 2015;37:218-229. doi:10.1016/j.biomaterials.2014.10.015

9. Bharadwaz A, Jayasuriya AC. Recent trends in the application of widely used natural and synthetic polymer nanocomposites in bone tissue regeneration. Mater Sci Eng C Mater Biol Appl. 2020;110:110698-110728. doi:10.1016/j.msec.2020.110698

10. Qin J, Zhong Z, Ma J. Biomimetic synthesis of hybrid hydroxyapatite nanoparticles using nanogel template for controlled release of bovine serum albumin. Mater Sci Eng C. 2016;62:377-383. doi:10.1016/j. msec.2016.01.088

11. Dwivedi C, Pandey I, Pandey $\mathrm{H}$, et al. In vivo diabetic wound healing with nanofibrous scaffolds modified with gentamicin and recombinant human epidermal growth factor. $J$ Biomed Mater Res A. 2018;106(3):641-651. doi:10.1002/jbm.a.36268

12. Dwivedi C, Pandey $\mathrm{H}$, Pandey AC, et al. In vivo biocompatibility of electrospun biodegradable dual carrier (antibiotic + growth factor) in a mouse model-implications for rapid wound healing. Pharmaceutics. 2019;11(4):180-197. doi:10.3390/pharmaceutics11040180

13. Singh AV, Dad Ansari MH, Dayan CB, et al. Multifunctional magnetic hairbot for untethered osteogenesis, ultrasound contrast imaging and drug delivery. Biomaterials. 2019;219:119394. doi:10.1016/j. biomaterials.2019.119394

14. Rahmani A, Hashemi-Najafabadi S, Eslaminejad MB, et al. The effect of modified electrospun PCL-nHA-nZnO scaffolds on osteogenesis and angiogenesis. J Biomed Mater Res A. 2019;107 (9):2040-2052. doi:10.1002/jbm.a.36717

15. Ghorbani FM, Kaffashi B, Shokrollahi P, et al. PCL/chitosan/Zndoped nHA electrospun nanocomposite scaffold promotes adipose derived stem cells adhesion and proliferation. Carbohydr Polym. 2015;118:133-142. doi:10.1016/j.carbpol.2014.10.071

16. Ji X, Yuan X, Ma L, et al. Mesenchymal stem cell-loaded thermosensitive hydroxypropyl chitin hydrogel combined with a three-dimensionalprinted poly( $\varepsilon$-caprolactone) /nano-hydroxyapatite scaffold to repair bone defects via osteogenesis, angiogenesis and immunomodulation. Theranostics. 2020;10(2):725-740. doi:10.7150/thno.39167

17. Shen MJ, Wang GG, Wang YZ, et al. Nell-1 enhances osteogenic differentiation of pre-osteoblasts on titanium surfaces via the MAPK-ERK signaling pathway. Cell Physiol Biochem. 2018;50 (4):1522-1534. doi:10.1159/000494651

18. Zhang X, Zara J, Siu RK, et al. The role of NELL-1, a growth factor associated with craniosynostosis, in promoting bone regeneration. J Dent Res. 2010;89:865-878. doi:10.1177/0022034510376401 
19. Li Y, Song H, Xiong S, et al. Chitosan-stabilized bovine serum albumin nanoparticles having ability to control the release of NELL-1 protein. Int $J$ Biol Macromol. 2018;109:672-680. doi:10.1016/j.ijbiomac.2017.12.104

20. Wang C, Hou W, Guo X, et al. Two-phase electrospinning to incorporate growth factors loaded chitosan nanoparticles into electrospun fibrous scaffolds for bioactivity retention and cartilage regeneration. Mater Sci Eng C. 2017;79:507-515. doi:10.1016/j.msec.2017.05.075

21. Hassan MI, Sultana N. Characterization, drug loading and antibacterial activity of nanohydroxyapatite/polycaprolactone (nHA/PCL) electrospun membrane. 3 Biotech. 2017;7:1-9. doi:10.1007/s13205017-0889-0

22. Wutticharoenmongkol P, Pavasant P, Supaphol P. Osteoblastic phenotype expression of MC3T3-E1 cultured on electrospun polycaprolactone fiber mats filled with hydroxyapatite nanoparticles. Biomacromolecules. 2007;8(8):2602-2610. doi:10.1021/bm700451p

23. Chen Y, Shafiq M, Liu M, et al. Advanced fabrication for electrospun three-dimensional nanofiber aerogels and scaffolds. Bioact Mater. 2020;5(4):963-979. doi:10.1016/j.bioactmat.2020.06.023

24. Xie J, Zhong S, Ma B, et al. Controlled biomineralization of electrospun poly( $\varepsilon$-caprolactone) fibers to enhance their mechanical properties. Acta Biomater. 2013;9(3):5698-5707. doi:10.1016/j.actbio.2012.10.042

25. Shamaz BH, Anitha A, Vijayamohan M, et al. Relevance of fiber integrated gelatin-nanohydroxyapatite composite scaffold for bone tissue regeneration. Nanotechnology. 2015;26(40):405101-405116. doi:10.1088/0957-4484/26/40/405101

26. Takebe J, Itoh S, Okada J, Ishibashi K. Anodic oxidation and hydrothermal treatment of titanium results in a surface that causes increased attachment and altered cytoskeletal morphology of rat bone marrow stromal cells in vitro. J Biomed Mater Res. 2000;51 (3):398-407.

27. Bhattacharjee P, Naskar D, Maiti TK, et al. Non-mulberry silk fibroin grafted poly (Ie-caprolactone)/nano hydroxyapatite nanofibrous scaffold for dual growth factor delivery to promote bone regeneration. $J$ Colloid Interface Sci. 2016;472:16-33. doi:10.1016/j.jcis.2016.03.020

28. Mathews S, Gupta PK, Bhonde R, et al. Chitosan enhances mineralization during osteoblast differentiation of human bone marrow-derived mesenchymal stem cells, by upregulating the associated genes. Cell Prolif. 2011;44:537-549. doi:10.1111/j.1365-2184.2011.00788.x

29. Aghaloo T, Cowan CM, Chou YF, et al. Nell-1-induced bone regeneration in calvarial defects. Am J Pathol. 2006;169:903-915. doi:10.2353/ajpath.2006.051210

30. Zhang X, Ting K, Bessette CM, et al. Nell-1, a key functional mediator of Runx2, partially rescues calvarial defects in Runx $2 \pm$ mice. J Bone Miner Res. 2011;26:777-791. doi:10.1002/jbmr.267

31. Jiang S, Lv LP, Landfester K, et al. Nanocontainers in and onto nanofibers. Acc Chem Res. 2016;49(5):816-823. doi:10.1021/acs. accounts. 5 b00524

32. Singh AV, Maharjan RS, Kanase A, et al. Machine-learning-based approach to decode the influence of nanomaterial properties on their interaction with cells. ACS Appl Mater Interfaces. 2021;13 (1):1943-1955. doi:10.1021/acsami.0c18470
33. Singh AV, Rosenkranz D, Ansari MHD, et al. Artificial intelligence and machine learning empower advanced biomedical material design to toxicity prediction. Adv Intell Syst. 2020;2:2000084. doi:10.1002/ aisy. 202000084

34. Hasan AS, Socha M, Lamprecht A, et al. Effect of the microencapsulation of nanoparticles on the reduction of burst release. Int J Pharm. 2007;344:53-61. doi:10.1016/j.ijpharm.2007.05.066

35. Ortega-Oller I, Padial-Molina M, Galindo-Moreno P, et al. Bone regeneration from PLGA micro-nanoparticles. Biomed Res Int. 2015;2015:415289. doi:10.1155/2015/415289

36. Çakır-özkan N, Eğri S, Bekar E, et al. The use of sequential VEGFand BMP2-releasing biodegradable scaffolds in rabbit mandibular defects. $J$ Oral Maxillofac Surg. 2017;75:221.e1-221.e14. doi:10.1016/j.joms.2016.08.020

37. Singh AV. Commentary on "peptide-conjugated nanoparticles as targeted anti-angiogenesis therapeutic and diagnostic in cancer" by Shaker A. Mousa, pharmaceutical research institute, Albany college of pharmacy and health sciences, Rensselaer, NY 12144, United States - peptide-conjugated nanoparticles for multimodal nanomedicine. Curr Med Chem. 2020;27(17):2927-2928. doi:10.2174/ 092986732717200604120627

38. Leibrock L, Wagener S, Singh AV, et al. Nanoparticle induced barrier function assessment at liquid-liquid and air-liquid interface in novel human lung epithelia cell lines. Toxicol Res. 2019;8(6):1016-1027. doi:10.1039/c9tx00179d

39. Ma S, Wang Z, Guo Y, et al. Enhanced osteoinduction of electrospun scaffolds with assemblies of hematite nanoparticles as a bioactive interface. Int J Nanomedicine. 2019;14:1051-1068. doi:10.2147/IJN. S185122

40. Zhang H, Mao X, Du Z, et al. Three dimensional printed macroporous polylactic acid/hydroxyapatite composite scaffolds for promoting bone formation in a criticalsize rat calvarial defect model. Sci Technol Adv Mater. 2016;17(1):136-148. doi:10.1080/14686996.2016.1145532

41. Sedghi R, Shaabani A, Sayyari N. Electrospun triazole-based chitosan nanofibers as a novel scaffold for bone tissue repair and regeneration. Carbohydr Polym. 2020;230:115707. doi:10.1016/j. carbpol.2019.115707

42. Bokui N, Otani T, Igarashi K, et al. Involvement of MAPK signaling molecules and Runx2 in the NELL1-induced osteoblastic differentiation. FEBS Lett. 2008;582(2):365-371. doi:10.1016/j. febslet.2007.12.006

43. Liu F, Wang X, Chen T, et al. Hydroxyapatite/silver electrospun fibers for anti-infection and osteoinduction. $J$ Adv Res. 2019;21:91-102. doi:10.1016/j.jare.2019.10.002

44. Tian X, Wang Q, Wu J, et al. Interaction of Nel-like molecule 1 with apoptosis related protein 3 with its influence on human dental pulp cells proliferation and differentiation into odontoblasts. Biochem Biophys Res Commun. 2019;518(2):246-252. doi:10.1016/j. bbrc.2019.08.042
International Journal of Nanomedicine

\section{Publish your work in this journal}

The International Journal of Nanomedicine is an international, peerreviewed journal focusing on the application of nanotechnology in diagnostics, therapeutics, and drug delivery systems throughout the biomedical field. This journal is indexed on PubMed Central, MedLine, CAS, SciSearch ${ }^{\mathbb{R}}$, Current Contents ${ }^{\mathbb{R}} /$ Clinical Medicine, $^{2}$
Journal Citation Reports/Science Edition, EMBase, Scopus and the Elsevier Bibliographic databases. The manuscript management system is completely online and includes a very quick and fair peer-review system, which is all easy to use. Visit http://www.dovepress.com/ testimonials.php to read real quotes from published authors. 